\title{
Deep brain stimulation for severe autism: from pathophysiology to procedure
}

\author{
Saurabh Sinha, BS, ${ }^{1}$ Robert A. McGovern, MD, ${ }^{2}$ and Sameer A. Sheth, MD, PhD ${ }^{2}$
}

1Department of Neurosurgery, Rutgers Robert Wood Johnson Medical School, New Brunswick, New Jersey; and ${ }^{2}$ Department of Neurological Surgery, Columbia University Medical Center, New York, New York

\begin{abstract}
Autism is a heterogeneous neurodevelopmental disorder characterized by early-onset impairment in social interaction and communication and by repetitive, restricted behaviors and interests. Because the degree of impairment may vary, a spectrum of clinical manifestations exists. Severe autism is characterized by complete lack of language development and potentially life-threatening self-injurious behavior, the latter of which may be refractory to medical therapy and devastating for affected individuals and their caretakers. New treatment strategies are therefore needed. Here, the authors propose deep brain stimulation (DBS) of the basolateral nucleus of the amygdala (BLA) as a therapeutic intervention to treat severe autism. The authors review recent developments in the understanding of the pathophysiology of autism. Specifically, they describe the genetic and environmental alterations that affect neurodevelopment. The authors also highlight the resultant microstructural, macrostructural, and functional abnormalities that emerge during brain development, which create a pattern of dysfunctional neural networks involved in socioemotional processing. They then discuss how these findings implicate the BLA as a key node in the pathophysiology of autism and review a reported case of BLA DBS for treatment of severe autism. Much progress has been made in recent years in understanding the pathophysiology of autism. The BLA represents a logical neurosurgical target for treating severe autism. Further study is needed that considers mechanistic and operative challenges.
\end{abstract}

http://thejns.org/doi/abs/10.3171/2015.3.FOCUS1548

KEY WORDS deep brain stimulation; autism; autistic spectrum disorder; amygdala; basolateral nucleus; neurodevelopmental disorders

A UTISM is a group of neurodevelopmental disorders affecting social cognition and behavior. It has a worldwide prevalence of $1 \%, 5^{50}$ and current estimates place the incidence of these disorders as high as 1 in 57 children in some regions. ${ }^{95}$ Males are disproportionately affected, with male-to-female ratios reported as high as 3 to $1{ }^{42,49}$ The Diagnostic and Statistical Manual of Mental Disorders, Fifth Edition (DSM-5), adopts a dyadic definition of core symptoms: 1) early-onset difficulties in social interaction and communication, and 2) repetitive, restricted behaviors and interests. ${ }^{41,50}$ Implicit in the DSM5 label for these conditions - autism spectrum disorderis their heterogeneity. At one end of this spectrum, individuals with autism are high functioning, enabling them to live on their own and maintain employment. Individuals characterized as low functioning, however, exhibit more severe symptoms. Numerous case reports documenting clinical courses consistently note that low-functioning individuals are nonverbal at baseline and exhibit poor social communication, self-injurious behavior (SIB), tantrums, and aggression that can be potentially life threatening to such patients and their caretakers..$^{1,12,52}$

For the latter presentation, medical management of the disorder typically involves antipsychotics, sedatives, $\alpha 2-$ adrenergic agonists, mood stabilizers, or anticonvulsants for behavioral control. ${ }^{1,85}$ However, for a subset of patients, these measures may still prove ineffective, and their symptoms may be refractory even to maximal medical therapy. In general, $35 \%-50 \%$ of people with autism engage in SIB ${ }^{72}$ but one study found that $32.1 \%$ of 135 consecutive patients with autism had refractory SIB. ${ }^{1}$ For these individuals, no other treatment options exist, and the severity

ABBREVIATIONS AF = arcuate fasciculus; $\mathrm{BLA}=$ basolateral nucleus of the amygdala; $\mathrm{DBS}=$ deep brain stimulation; $\mathrm{DMN}=$ default mode network; ECoG = electrocorticography; $E E G$ = electroencephalography; $\mathrm{fMRI}=$ functional $\mathrm{MRI} ; \mathrm{mPFC}=$ medial $\mathrm{PFC} ; \mathrm{OCD}=$ obsessive-compulsive disorder; $\mathrm{PFC}=$ prefrontal cortex; $\mathrm{SIB}=$ self-injurious behavior; UF = uncinate fasciculus.

SUBMITTED February 3, 2015. ACCEPTED March 26, 2015.

INCLUDE WHEN CITING DOI: 10.3171/2015.3.FOCUS1548.

DISCLOSURE The authors report no conflict of interest concerning the materials or methods used in this study or the findings specified in this paper. 
of their condition can lead to significant distress, including constant care, multiple comorbidities, and decreased quality of life. ${ }^{1,50}$ In such cases, neurosurgical intervention in the form of deep brain stimulation (DBS) may be a viable therapeutic option. Although application of DBS was pioneered in the treatment of movement disorders, its indications have continued to expand within both neurological and psychiatric diseases, including depression ${ }^{39}$ and obsessive-compulsive disorder (OCD) ${ }^{37}$ In fact, use of DBS for severe autism has been previously reported in 3 patients with some success ${ }^{86,88}$ although it has not been otherwise significantly discussed in the literature.

Here, we expand on the discussion of DBS for treating severe autism by examining the latest developments in characterizing the underlying pathophysiology of autism, including increasingly well-characterized neural networks that exhibit dysfunction in individuals with this disorder. We discuss the structural and functional pathologies with special consideration of amygdalar dysfunction in autism. Lastly, we synthesize the current understanding of autism pathophysiology by proposing the basolateral nucleus of the amygdala (BLA) as a target for DBS for severe autism.

\section{The Pathophysiology of Autism Spectrum Disorder \\ Etiological Considerations: Genetic and Environmental Impact on Microstructure}

Autism was originally described in 1943 by Leo Kanner, who recognized characteristic social deficits in a group of children. ${ }^{45}$ Our understanding of the disease course and pathogenesis of autism has grown markedly since that time, and funding for autism research has considerably increased in the last 2 decades.$^{50}$ Contextually, it is important to note that most of these studies have been performed in high-functioning individuals. Common themes have emerged, however. Etiologically, autism is broadly multifactorial, including genetic and environmental influences that have been studied in increasing detail. In particular, studies of gene mutations associated with autism suggest that these mutations alter developmental pathways involved in synaptogenesis and axonal extension and motility. 19,31,93 Postmortem studies support the role of these mutations in creating altered neuronal and axonal structure. For example, a recent study assessing neocortical neurons in patients with autism found consistent but heterogeneous patches of neuronal disorganization in prefrontal and temporal cortices ${ }^{87}$ Moreover, increases in the ratio of smalldiameter to large-diameter axons have been reported in regions of the prefrontal cortex (PFC) such as the anterior cingulate cortex (ACC).$^{97}$ Other postmortem studies have indicated bidirectional changes in neuron size depending on location (for example, smaller neurons in limbic system structures) and increased amygdalar and hippocampal cell density. ${ }^{10,66,71}$

Taken together, these findings suggest that both genetic mutations and environmental insults are associated with the microstructural changes observed in individuals with autism. These changes also appear to underlie gross structural alterations that ultimately have implications for the characteristic neural network dysfunction underlying autism. Along with the core symptoms of autism, these dysfunctional networks may also give rise to neurological and psychiatric comorbidities that affect up to $80 \%$ of individuals with autism (Fig. 1). ${ }^{50}$

\section{Socioemotional Processing: Current Theory}

An important trend in structural and functional studies of the brain in autism is the involvement of regions mediating socioemotional processing. Socioemotional processing, also termed social cognition, is broadly defined as the processing and storage of information necessary to navigate social contexts. ${ }^{14,91}$ As such, it is prudent to review current ideas about neural networks involved in socioemotional processing.

Because socioemotional processing depends on many intellectual abilities, such as memory, emotion, language, and mentalization (thinking about what others are thinking), it necessarily involves activities in a number of brain regions. Recent work has identified 3 main components of what appears to be a network for socioemotional processing: 1) the limbic system, 2) the facial processing system, and 3 ) the mirror neuron network (Fig. 2). ${ }^{6,17}$ Current theory about the limbic system, derived from functional neuroimaging and lesion studies, delineates 3 frontotemporal circuits constituting a limbic network: the hippocampaldiencephalic and parahippocampal-retrosplenial circuit, the temporoamygdalo-orbitofrontal circuit, and the default mode network (DMN). ${ }^{17}$ These circuits work together in mediating the limbic system's central role in memory and emotion. The facial processing system consists of the amygdala, fusiform gyrus, and superior temporal sulcus.

Socially relevant cues abound in the face, including eye gaze, facial recognition, and facial expression. ${ }^{6}$ The facial processing system mediates these components of social cognition and communicates them to higher-order frontal, temporal, and occipital centers. The mirror neuron system, which mediates higher-order social cognition, is located at the temporoparietal junction and communicates anteriorly with the medial PFC (mPFC). ${ }^{91}$ This network processes inferences about intentions of self and others based on action and receives input from visual, premotor, and association cortices. The $\mathrm{mPFC}$ receives this input, along with the dorsolateral PFC and superior temporal sulcus, to integrate social information over time for longterm processing. ${ }^{91}$ Together, the structures of the mirror neuron network facilitate "theory-of-mind" (or mentalizing) processes, ${ }^{30}$ which are involved in acquiring insight into the mental state of others. ${ }^{9}$

The limbic system, facial processing system, and mirror neuron network are connected by a number of white matter tracts. The uncinate fasciculus (UF) is the major white matter tract connecting the amygdala and other anterior temporal lobe structures with PFC regions. ${ }^{6,48}$ The arcuate fasciculus (AF) connects frontotemporoparietal perisylvian regions and is involved notably in speech and language networks and in the mirror neuron network. ${ }^{6}$ The cingulum bundle, a white matter tract underlying the cingulate gyrus, connects cingulate cortex regions with numerous cortical regions, including the frontal, temporal, parietal, and occipital cortices. Importantly, it facilitates widespread connection to limbic system structures (again, 


\section{Genetic and Environmental Influences}
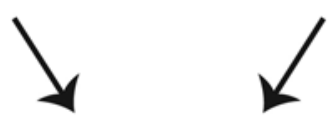

\section{Impaired Neurodevelopment}<smiles>C[13CH]</smiles>
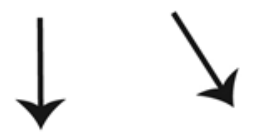

\section{Microstructural Defects \\ - Cell density changes \\ - Fewer large diameter axons}

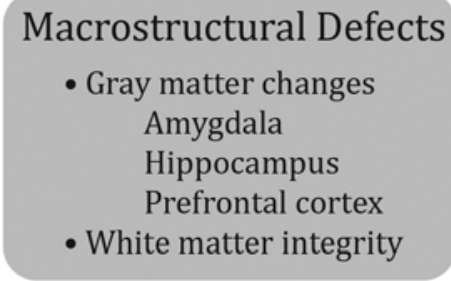

-White matter integrity

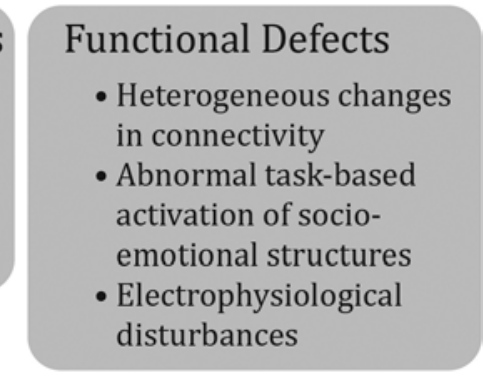

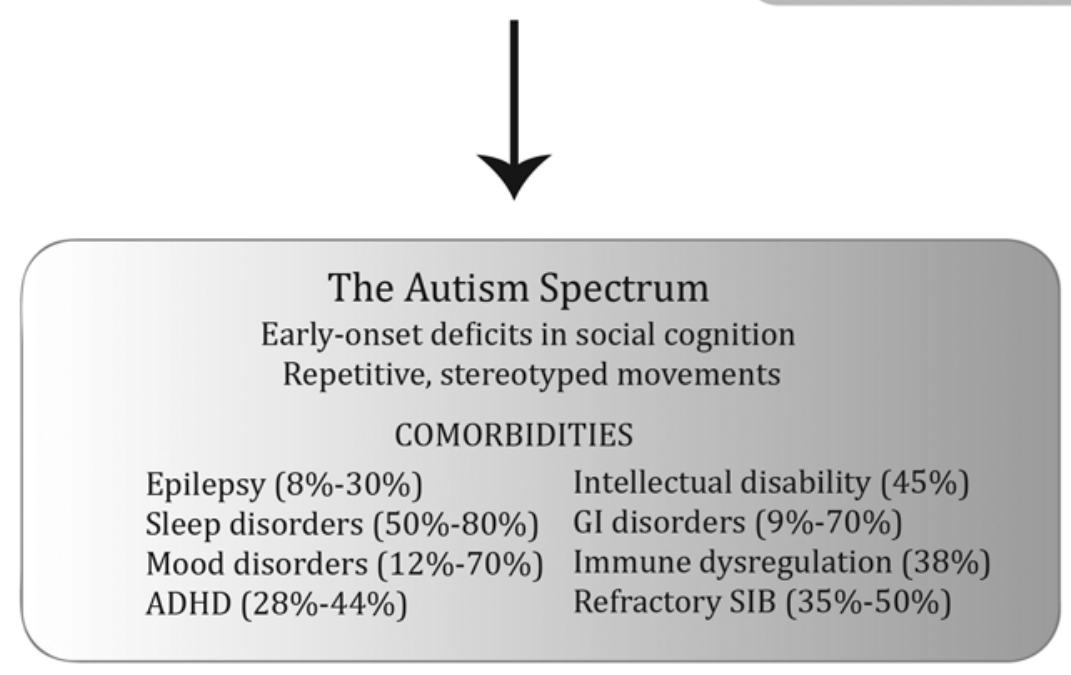

FIG. 1. A schematic representation of the pathophysiology of autism. Genetic and environmental insults converge on neurodevelopmental pathways, including those involved in synaptogenesis and axon motility. At progressively larger scales, these insults create heterogeneous patterns of microstructural, macrostructural, and functional defects that consistently involve structures playing a role in socioemotional processing. Together, these abnormalities give rise to the spectrum of clinical manifestations of autism and its many associated comorbidities. $\mathrm{ADHD}=$ attention deficit-hyperactivity disorder; $\mathrm{GI}=$ gastrointestinal; mood disorders = major depressive and bipolar disorders and OCD.

such as the amygdala), and to the mirror neuron network structures. ${ }^{6,38}$ Other important white matter tracts involved in socioemotional processing include the inferior longitudinal fasciculus and inferior frontoorbital fasciculus. ${ }^{6}$

Thus, socioemotional processing, or social cognition, is a wide-ranging process with both lower-level and higher-level components across numerous regions. It involves complex short- and long-range inputs and outputs into structures across 3 major components of a socioemotional network. As autism is partly a disorder of social cognition, it is logical that abnormal structure and function of these regions should exist in individuals with this disorder. Indeed, structural and functional neuroimaging studies have repeatedly shown abnormalities in nodes of these networks and their associated white matter tracts, which we discuss below.

\section{Structural Pathology}

Recent meta-analyses have begun to synthesize several disparate findings in structural studies of autism. These heterogeneous findings are likely due to the heterogeneity of the disease symptoms and to differences in disease 

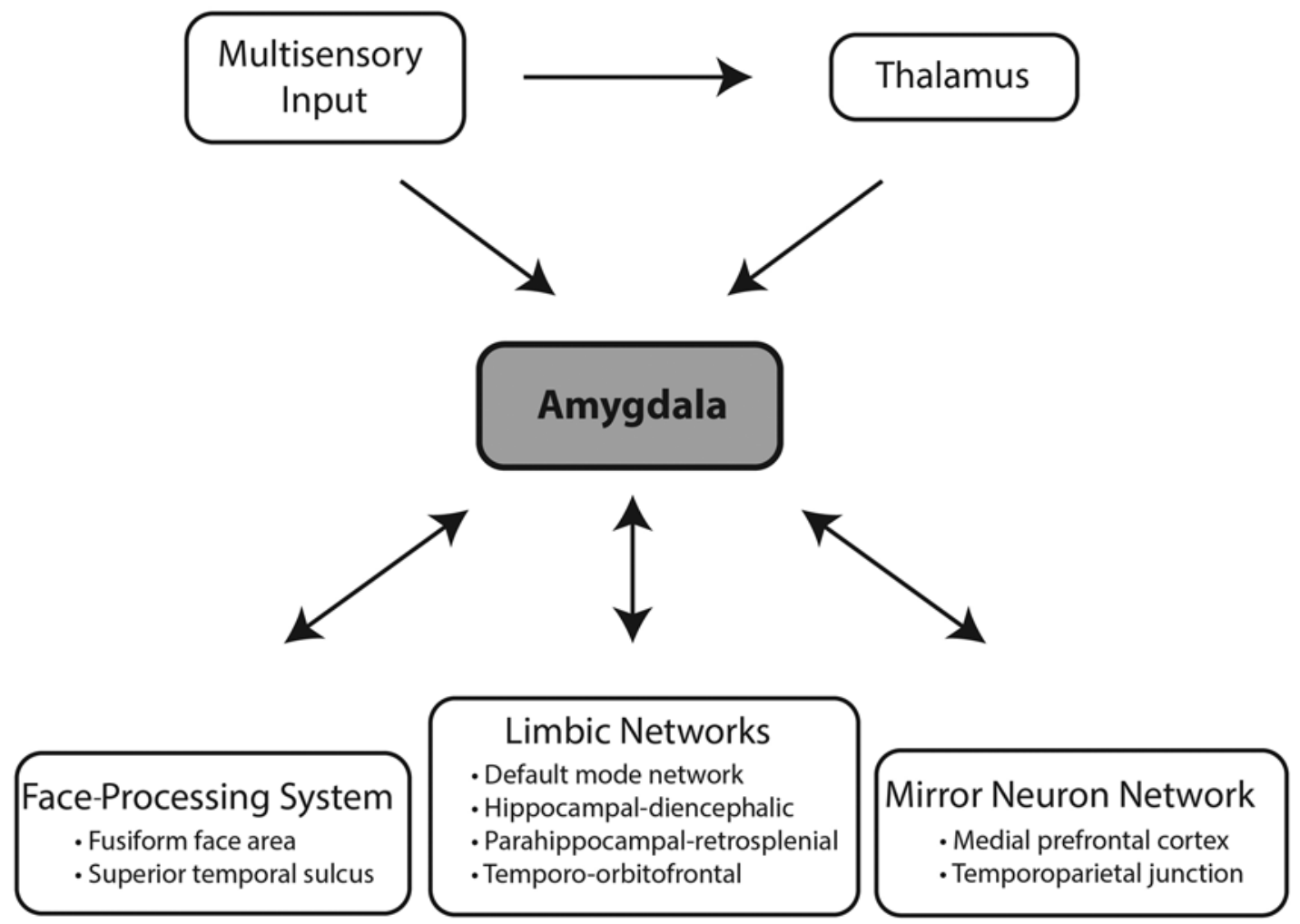

FIG. 2. The central role of the amygdala in socioemotional processing. Among other inputs (see text), the amygdala receives sensory input across all modalities from the thalamus and from primary sensory cortices. These stimuli may contain information pertinent to social cognition, such as speech, faces, and smell. As the amygdala is a key component of multiple networks involved in socioemotional processing (including the limbic network, face-processing system, and mirror neuron network), the routing of sensory information through the amygdala appears to be crucial for proper integration in the social domain. The face-processing system mediates the processing of eye gaze and facial recognition, among other socially relevant facial cues. The limbic network consists of 3 frontotemporal circuits governing memory and emotion. The mirror neuron network mediates higher-order social cognition, including processing the intentions of others. Amygdalar impairment results in social deficits consistent with abnormalities in these networks and has been implicated as an important contributor to the pathophysiology of autism.

severity. Despite these concerns, reliable differences in structures involved in socioemotional processing have been described in individuals with autism.

One robust trend identified in structural studies is early overgrowth of both cortical and subcortical regions involved in socioemotional processing as measured by tissue thickness and surface area. The amygdala, part of the previously described limbic and facial processing systems, is a particularly well-characterized region exhibiting this trend. In a longitudinal study, children with autism who were between 7.5 and 12.5 years of age exhibited amygdalar enlargement compared with typically developing children. ${ }^{83}$ During this period, overall amygdalar volume was greater in children with autism than in the controls.

Normal amygdalar development typically consists of an accelerated period of growth from preadolescence until age 18 years. With the bulk of amygdalar growth happening far earlier in individuals with autism followed by a period of slowed growth, a growth trend opposite of that in controls, amygdalar volume tends to normalize in individuals with autism by age $188^{83}$ This abnormal growth trajectory suggests a completely different neurodevelopmental sequence in autism. Importantly, the specific involvement of the amygdala, which is integral to multiple socioemotional processing circuits, is crucial and bears on our further discussion. As reported in a recent meta-analysis, ${ }^{26}$ other regions involved in socioemotional processing that also exhibit early overgrowth include the insula and cingulate gyrus.

Abnormal growth patterns are also observed in other structures involved in socioemotional processing. The hippocampus, also a component of the limbic system, exhibits increased volume in both children and adults with autism compared with controls, indicating consistent overgrowth. ${ }^{83}$ Similarly, prefrontal and anterior temporal gray matters also show increased volume. ${ }^{27}$ Moreover, these volumetric differences are associated with differences in the severity of social and behavioral symptom domains of autism; that is, the more drastic the volume change, the more severe the phenotype. It should be noted, however, that all subjects in the latter study were male. Given recent studies suggesting differences between the sexes in the neural architecture of autism, ${ }^{51}$ it is difficult to discern whether these conclusions extend to female individuals as well.

Along with the dysfunctional nodes of socioemotional networks described above, impaired integrity of white matter tracts involved in socioemotional processing has 
also been reported in studies using diffusion tensor imaging. ${ }^{6}$ Decreases in structural integrity (measured by fractional anisotropy) and volume (measured by number of streamlines) of the UF, cingulum bundle, and AF have been demonstrated and have been associated with autism symptoms. ${ }^{18,70}$ Taken together, these findings suggest altered neurodevelopmental dynamics in autism that are likely related to the aforementioned underlying microstructural alterations. Moreover, the specific regions involved appear to be closely related to a socioemotional network, which may explain the social deficits displayed by individuals with autism. Indeed, as we will discuss below, functional studies support this trend.

\section{Functional Pathology}

In recent years, functional neuroimaging studies have begun to provide a detailed explanation of the pathophysiological networks of autism. These studies have assessed specific structures within brain networks by measuring activation in response to tasks. They have also assessed connectivity by mapping regions that are synchronously activated. ${ }^{9}$ In the latter pursuit, studies have supported the existence of widespread decreased connectivity among cortical networks, particularly among socioemotional regions in individuals with autism. These studies have provided evidence for what was originally posited as "weak central coherence" theory, which explains why the cognitive processes of autism are centered on detail rather than on the global picture. ${ }^{68}$

This endeavor was recently brought to its largest scale yet with the Autism Brain Information Data Exchange (ABIDE), a collaborative effort sampling neuroimaging and phenotypic data from $>500$ male patients with autism. Using resting-state MRI data to assess network connectivity, the first study with this database confirmed previous findings of cortico-cortical and interhemispheric network underconnectivity. ${ }^{25}$ Similar trends of underconnectivity have been reported for other limbic, somatosensory, and motor networks as well..$^{33}$ Combining these findings with those of task-based functional MRI (fMRI), functional neuroimaging studies agree with structural studies about the crucial role of socioemotional networks in the pathophysiology of autism. The underlying principle of global underconnectivity and resultant changes in activation in people with autism is that, once encoded, stimuli are processed atypically within dysfunctional networks, which, importantly, include socioemotional networks. Poor integration of information during processing and during communication with downstream brain regions may then ultimately lead to the clinical presentation of autism. Heterogeneity in the creation of these dysfunctional networks during development likely contributes to phenotypic heterogeneity. With this basic framework, we discuss relevant specific functional findings in the socioemotional networks described above.

\section{Socioemotional Network Dysfunction}

A recent meta-analysis of functional imaging studies assessing responses to mentalization and to emotional processing tasks noted decreased activation of socioemotional regions such as the pregenual $\mathrm{ACC}, \mathrm{mPFC}$, and amygdala. ${ }^{24}$ Importantly, these regions are overlapping structures within the 3 aforementioned socioemotional networks (that is, the limbic system, face-processing system, and mirror neuron network). In controls, their coactivation on multiple tasks related to social cognition indicates their wider connectivity as facilitated by white matter tracts such as the UF, cingulum bundle, and AF. In patients with autism, their hypoactivity recapitulates abnormalities observed in structural studies demonstrating volumetric changes and impaired white matter tract integrity. Other studies have looked separately at individual components of these socioemotional networks.

For example, the DMN is 1 of the 3 circuits proposed to contribute to limbic system function. Anatomically, the DMN extends from the ACC and mPFC anteriorly to the posterior cingulate cortex and precuneus posteriorly. ${ }^{17}$ Studies of the DMN have determined that it is functionally active during "resting state," which has been construed as an introspective process. ${ }^{17}$ To assess activity in the DMN, a study subject is instructed to relax rather than to attend to stimuli. The resultant change in attentional state appears to rely on DMN activation.? During goal-directed behavior, however, synchronous DMN deactivation appears to be a crucial step in attending to and executing tasks, mentalizing, and in engaging working memory. ${ }^{17}$ Individuals with autism are impaired in deactivating the DMN during cognitive tasks, ${ }^{47}$ suggesting dysfunction in breaking introspection to attend to stimuli. Because attending to relevant stimuli is crucial for social cognition, and given the DMN's connections to other socioemotional structures through the UF, cingulum bundle, and the fornix, ${ }^{6} \mathrm{DMN}$ dysfunction could give rise to autism phenotypes.

The face-processing system, another component of the socioemotional network, has been studied extensively in people with autism. A recent study demonstrated hypoactivation of visuoperceptual regions in response to a facial recognition task. In particular, the degree of hypoactivation in the fusiform face area and in the superior temporal sulcus appears to track with symptom severity. ${ }^{80}$ Heterogeneous abnormalities in amygdalar activation are also observed, although a recent study notes that these differences may be the result of the forms of stimuli being shown. ${ }^{80}$ Specifically, amygdalar hypoactivation ${ }^{8,35}$ is typically observed when individuals with autism compare affective and neutral faces to objects, whereas amygdalar hyperactivation ${ }^{22,90}$ is observed when they compare affective and neutral faces to simple fixation points. Evidently, amygdalar activation patterns are dependent on context. However, it is important to note that differences in amygdalar activation along with hypoactivity throughout other facial processing regions suggest that individuals with autism have functional abnormalities in face processing, manifesting as poor eye contact, gaze disturbances, and as impaired affective recognition of faces.

Although not specifically processed by any one particular component within the socioemotional processing framework, problem solving is another important aspect of social cognition. Problem solving engages similar brain regions as are involved in socioemotional processing. Moreover, just as socioemotional processing depends on different domains of sensory input (that is, visual processes for faces and auditory processes for speech), problem 
solving proceeds through multiple domains. Visual and verbal domains are 2 such examples. In control subjects, verbal problems are typically processed separately from visuospatial problems and primarily engage frontotemporal language networks. People with autism, however, display preferential use of visuospatial reasoning for solving both visual and verbal problems. ${ }^{61}$ Neuroimaging studies during visual and verbal tasks have shown that occipitoparietal-visuospatial networks are preferentially activated over frontotemporal language networks in individuals with autism. ${ }^{77}$ Connectivity studies in the same subjects with autism also demonstrated increased connectivity in posterior visuospatial networks but decreased connectivity in anterior, frontotemporal networks, a trend opposite of that seen in controls. ${ }^{77}$

Functional studies on autism thus corroborate structural studies implicating socioemotional networks as underlying the pathophysiology of autism. Both task-based and resting-state connectivity studies indicate patterns of abnormal connectivity in these regions. Interestingly, just as structural heterogeneity exists in autism, functional heterogeneity exists as well. In some individuals, certain networks are overconnected, whereas in others, these networks may be underconnected. A recent study notes that this differential connectivity may in fact be representative of person-to-person variation in network distortion from the norm. ${ }^{36}$ However, the important common theme emerging from these studies is consistent abnormality of the socioemotional networks. Because these networks ultimately give rise to electrical activity, electrophysiological abnormalities are to be expected, and have indeed been demonstrated.

\section{Electrophysiological Disturbances}

Altered excitatory or inhibitory control is implicated in the abnormality in the electrophysiological domain of autism pathophysiology. Genetic studies in autism have identified mutations in genes encoding $\gamma$-aminobutyric acid (GABA) receptor subtypes. This trend is borne out in postmortem studies, which have noted decreases in the densities of $\mathrm{GABA}_{\mathrm{A}}$ and $\mathrm{GABA}_{\mathrm{B}}$ receptors in individuals with autism. Along with the proposed involvement of glutamatergic neurotransmitter systems, ${ }^{15,59}$ these findings suggest altered excitatory or inhibitory control shifting toward excitatory activity, which manifests as increased abnormal oscillatory activity across the brain. ${ }^{74}$ When combined with dysfunctional connectivity, this sets the stage for abnormal electrophysiological signals ${ }^{69}$ Such signals have been reported from studies using electroencephalography (EEG) and magnetoencephalography; studies of electrophysiology in autism using more invasive techniques such as electrocorticography (ECoG) and singleneuron measurements are scarce.

Resting-state spectral analyses of EEGs in patients with autism have revealed increases in midline gamma ${ }^{65}$ and posterior thet $\mathrm{a}^{20}$ power, although these findings have been variable. A recent magnetoencephalography study comparing brain activity in individuals with autism with that in age-matched controls indicated that those with autism have increased resting-state power of multiple frequency bands. ${ }^{21}$ Alpha and delta powers were elevated in frontopa- rietal channels, and increases in the latter have been associated with gray-white matter disconnection..$^{40}$ In the study by Cornew and colleagues, ${ }^{21}$ broadband gamma power was increased in posterior channels. Stimulus-induced electrophysiological changes in autism are not well studied, but EEG studies of pure-tone auditory stimuli in individuals with autism have shown increased event-related potential latency along with deficits in phase-locked gammaband power in the superior temporal gyrus. ${ }^{28,69}$ Moreover, these oscillatory signals showed decreased coherence in response to the stimuli, indicating that oscillatory activity was more frequently out of phase in individuals with autism. Taken together, these results suggest that the dysfunctional networks of autism produce not only an abnormal resting state, but also abnormal responses to stimuli and that these abnormalities are detectable with electrophysiological methods.

Abnormalities in the gamma range in people with autism are particularly interesting because the gamma band is hypothesized to facilitate the integration of multiple features of a stimulus. ${ }^{13}$ For example, a given object has different features such as color, position, and smell. These features appear to synchronously stimulate different neurons within specific temporal windows in various cortical regions in the gamma band. The overall pattern of synchronized, phase-coupled activity represents a reconstruction of the stimuli in the brain..$^{13}$ This concept is referred to as temporal binding, and its instrumental phase-coupling appears to be mediated by long-range interneurons. ${ }^{44}$ In autism, abnormalities in gamma power, along with established changes in interneuron integrity, may ultimately underlie abnormal stimulus processing. Given the structural and functional abnormalities noted above, temporalbinding deficits, which have been proposed to characterize autism ${ }^{16}$ likely involve socioemotional networks. In highfunctioning autism, these deficits may be most pronounced at the higher level of the $\mathrm{mPFC},{ }^{58}$ but in low-functioning phenotypes, network pathology may be more severe in subcortical structures.

Because the amygdala, a more basal structure in socioemotional processing, has robustly shown abnormalities in autism, more severe amygdalar dysfunction may partly explain more severe phenotypes. Clinical manifestations such as SIB and the profound nonverbal nature of patients with severe autism may thus be a result of abnormal stimulus processing and multisensory integration in basal structures of social cognition.

\section{Summary}

Genetic studies of autism have identified mutations in complexes of genes involved in synaptogenesis and axon motility in those affected by this condition. These mutations interfere with typical neurodevelopment in utero through childhood. Microstructural, macrostructural, and functional analyses provide further support for these neurodevelopmental alterations, especially in key regions for socioemotional processing. Although these studies indicate variability in structural features, consistent with phenotypic heterogeneity, major trends are still apparent. Microstructurally, examples include an altered ratio of short- to long-diameter axons and disorganization of cortical layers. Macrostructurally, MRI studies of brain 
volume in individuals with autism and in age-matched controls have consistently shown cortical and subcortical gray matter overgrowth early in brain development, particularly involving frontal and temporal structures such as the PFC and the amygdala. Diffusion tensor imaging studies have demonstrated impaired integrity of white matter structures that are key for socioemotional processing, including the UF and AF.

Through resting-state fMRI, a narrative of widespread global underconnectivity has emerged, which is prominently evident in socioemotional networks. Moreover, task-based fMRI studies show decreased activation in networks involved in socioemotional processing. Lastly, electrophysiological studies indicate alterations of both resting-state and stimulus-induced oscillatory activities, notably in broadband gamma range, in people with autism. Taken together, these results suggest that neurodevelopmental alterations in autism involving socioemotional structures produce large-scale network dysfunction that is quantifiable with functional neuroimaging and electrophysiological techniques. It is this dysfunction that is thought to underlie the phenotypes of autism.

While many regions are involved in the processes detailed above, one structure in particular bears further discussion in the pathophysiology of autism: the amygdala. As we have noted, structural abnormalities of the amygdala have been consistently observed in individuals with autism, along with functional perturbations involving its associated socioemotional networks. We now turn to a more in-depth discussion of the role of the amygdala in social cognition and in autism pathophysiology. We then synthesize this information by discussing DBS of the BLA to treat severe autism.

\section{Role of the Amygdala in Social Cognition}

The amygdala, or the amygdaloid nuclear complex, is a set of nuclei located in the medial temporal lobe anterior to the hippocampal formation. ${ }^{76}$ It is a major component of the limbic system and is a well-characterized node within the affective loop of the parallel corticostriato-thalamocortical circuits..$^{5}$ Although the total number of nuclei and even the characterization of the amygdala as one structure has been debated, ${ }^{2}$ the 3 major groups of nuclei relevant for this discussion are the central, basolateral, and superficial nuclei. ${ }^{76}$ Each nucleus has different connections and different presumed functions. The BLA acts as a communication channel between the central and superficial nuclei and is reciprocally connected with the orbitofrontal cortex, ACC, and the mPFC. ${ }^{2,54}$ It also sends projections to the ventral striatum, which is an important node of the limbic system, while also receiving multi-sensory input. These connections highlight the amygdala as a node connecting sensory stimuli to higher-level areas of social cognition. The central nucleus of the amygdala serves as the major output to various regions, including the hypothalamus, thalamus, ventral tegmental area, and reticular formation. ${ }^{92}$ The superficial nucleus contains the nucleus of the lateral olfactory tract. ${ }^{76}$

Nonhuman primate electrophysiology studies, as well as lesion, fMRI, and limited ECoG studies in humans, have helped determine the role of the amygdala in cogni- tion. An important trend that has emerged from these studies is the important role of the amygdala in fundamental elements of social cognition. According to a recent metaanalysis of ECoG studies, ${ }^{62}$ the major functions ascribed to the amygdala are judgments of emotional valence of a stimulus, ${ }^{94}$ selective encoding and detection of relevant stimuli, ${ }^{78}$ familiarity versus novelty judgments of stimuli regardless of sensory modality, ${ }^{29,43}$ and the processing of social cues from the face (particularly from the eyes). ${ }^{60,79}$

Interestingly, the authors also note differential temporal windows within which these processes occur. The early window (50-290 msec) includes affective processing or the distinction of emotion within stimuli. Differential frequencies of elicited gamma-band activity are observed for aversive versus pleasant stimuli. The intermediate window (270-470 msec) includes detection of novel multisensory stimuli related to a current task. The late window (600$1400 \mathrm{msec}$ ) corresponds again to affective processing with additional top-down control from attentional and workingmemory processes. It is apparent that amygdala-evoked responses emphasize both the emotional components of stimuli-aversive versus pleasant, for example - and their social components as well, as evidenced by response to face and eyes. Given that these components may be processed in an early temporal window preceding conscious experience and executive control, but then processed again with top-down control, it is reasonable to view the amygdala as an instrumental node in social cognition.

The amygdala appears to play a role in reward learning as well.32 Early animal studies of the amygdala noted a fear-conditioning role, ${ }^{46,53}$ this concept has expanded to include a more complex role of evaluation of a stimulus within a social context. Indeed, multiple reports note that the amygdala may continuously monitor the biological value of stimuli, eventually imbuing relevant stimuli with specific emotional "labels."2,78 Given its apparent role in social cognition, this function likely centers around the valuation of social stimuli from all sensory modalities. These values are then transmitted to higher-order cortical centers for executive processing and, ultimately, for manifestation of behavior.

Although an in-depth review of amygdalar function ${ }^{2,62}$ is outside the scope of this article, it is important to note that amygdalar structure and function vary highly among individuals. This variation may be context dependent, shaped by the experience of the person. ${ }^{2}$ However, the amygdala does have a significant role in major components of social cognition (Fig. 2). Because robust amygdalar abnormalities have been identified in people with autism, it represents a structure of major importance in autism pathophysiology and, potentially, therapy.

\section{The Amygdala in Autism}

As previously discussed, in the abnormal neurodevelopment underlying autism, the frontal and temporal lobes are markedly affected. In particular, structural and functional perturbations of medial temporal lobe structures, including the amygdala, are well established. Here, we discuss further studies on the involvement of the amygdala in autism with particular emphasis on 2 major domains characterizing severe autism: social deficits and SIB. 


\section{Social Deficits}

Amygdalar lesion studies have indicated abnormal fear processing, ${ }^{3}$ impaired modulation of memory with emotional content, ${ }^{4}$ and abnormal reductions in eye gaze when looking at human faces. ${ }^{84}$ The latter finding has been shown at the single-neuron level in people with autism as well. Specifically, one study found groups of amygdalar neurons responsive to whole-face images both in people with autism and in controls. ${ }^{75}$ However, in neurons responsive to face regions, individuals with autism showed relative insensitivity to the eye region and greater sensitivity to the mouth region. Taken together, these findings indicate that symptoms overlap between individuals with amygdalar lesions and those with autism. However, numerous differences between these 2 groups have also been noted. ${ }^{96}$ Given the widespread network connectivity dysfunction characteristic of autism, these differences are not surprising and point to autism as a broad network disorder rather than one solely implicating the amygdala.

Imaging studies have robustly characterized amygdalar dysfunction in autism. When the structural and functional pathology of the amygdala is considered with its aforementioned normal role, it becomes apparent how amygdalar dysfunction could give rise to deficits in the multiple elements of social cognition. For example, eye gaze and face processing are 2 specific, well-defined functions of the amygdala, and its damage therefore affects both processes. In people with autism, these processes are among the features within the core domains of socioemotional processing that are affected,$^{50}$ manifesting as lack of eye contact and impaired ability to process social cues from faces.

Moreover, the amygdala's general role in affective valuation of biologically relevant stimuli provides a potential mechanistic link to the impaired social cognition observed in individuals with autism. Consider the importance of all sensory modalities in receiving and interpreting social cues: from the visual input of a person's face and social setting, to the auditory stimulus of a person's speech, to communication by touch, social information is embedded in the sensory experience. Insofar as social cognition is dependent on the proper valuation of crucial stimuli, amygdalar dysfunction could contribute to deficits in social cognition. Stated another way, if these stimuli are not processed properly by the amygdala, which relays to higher-order cortical centers, behavior is affected. The same principles may hold for the SIB observed in severe autism.

\section{Self-Injurious Behavior}

Self-injurious behavior is not a core feature of autism, but it can be debilitating for some people with autism and problematic for their caretakers. ${ }^{23}$ Studies analyzing the impact of SIB on children and adults with severe autism reported that the strength of physical strikes may approach that of a professional boxer at a rate of up to 80 per minute. ${ }^{63,64}$ This feature is not specific to autism and may be observed also in other forms of intellectual disability, such as Fragile X or Rett syndrome. ${ }^{23}$ Although the mechanism for SIB is not completely understood, one hypothesis is that aberrant sensory processing leads to abnormal judgments of stimuli as noxious. This aversive valuation leads to self- inflicted pain in an effort to engage inhibitory control from descending interneurons on the perceived noxious stimuli such that processing of these initial stimuli is shut down. As such, self-harm may be a coping mechanism for intolerable sensory experience in a person who cannot otherwise communicate; hence, it is associated with severe intellectual disability. ${ }^{67}$ These noxious sensory experiences could include benign but abnormally processed sensoria such as harmless sounds or truly noxious stimuli such as pain. In regard to the latter, some studies have reported a high rate of underlying undiagnosed medical conditions (for example, gastroesophageal reflux disease ${ }^{55}$ ) in patients with SIB; in some cases, correct diagnosis and treatment of these conditions was associated with a decrease in SIB. ${ }^{11}$ Other studies have observed similar trends of decreased SIB directed at a painful site after treatment of the cause of pain..$^{56}$

In autism, this intolerable sensory experience may be the result of altered sensitivity to stimuli, which can occur across all sensory domains, and in either direction (that is, hyper- or hyposensitivity). As with the core symptoms of autism, the severity of these alterations in sensitivity is heterogeneous ${ }^{57}$ Severe hypersensitivity to otherwise benign auditory stimuli may manifest behaviorally as shielding of the ears from the sound. Electrophysiological studies have shown alterations in latency of event-related potentials involved in early auditory processing $;{ }^{73}$ however, the influence of top-down cortical control may also play a role in these changes. ${ }^{57}$ Similarly, other sensory modalities, including vision and touch, have been shown to exhibit changes concordant with gross alterations in sensitivity. ${ }^{57}$ Importantly, hypersensitivity in processing has been linked to SIB. Thus, this behavior may represent a coping mechanism for what is felt as a noxious stimulus because of its perceived intensity.

The amygdala may be crucial in the formation of SIB because of its aforementioned role in stimulus valuation. Because it receives an integrated, multisensory signal and appears to encode emotional valence, the amygdala may be the initial point in the network where sensory experience is given an "affective label." Indeed, the amygdala has been described as a component of a "pain matrix" involved in processing pain. ${ }^{92}$ Moreover, the amygdala relays these signals to higher-order cortical centers to eventually affect behavior. Importantly, the amygdala, cortical centers, and the networks that connect them all exhibit some degrees of abnormality in persons with autism. Given this confluence of dysfunction, adjusting amygdalar signal through neuromodulation may represent a means of therapeutic relief for SIB that is otherwise refractory to treatment.

\section{Summary}

Two major symptom domains that may require treatment in severe autism are social deficits, including being nonverbal and nonresponsive to speech, and SIB, which can be life threatening. The amygdala seems to play an important role in the pathophysiology of these abnormalities. Broadly speaking, one of the normal roles of the amygdala is to evaluate biologically relevant stimuli in a continuously updated, context-dependent manner. Amygdalar dysfunction, on the other hand, is robustly associated 
with autism. Moreover, sensory processing is often altered in autism. Taken together, these findings indicate that, through abnormal stimulus valuation and through anomalous projections to higher-order cortical centers, amygdalar dysfunction may underlie the social deficits and SIB that characterize severe autism. As such, neuromodulation of amygdalar targets may represent a therapeutic intervention for patients in whom other therapies have failed.

\section{Role for DBS}

Deep brain stimulation was initially approved by the FDA in 1997 for treating essential tremor and in 2002 for alleviating Parkinson disease. Since then, its use has expanded to psychiatric disorders. In 2009, a humanitarian device exemption was given to DBS for refractory OCD, and studies have demonstrated its efficacy for this indication. ${ }^{37}$ Deep brain stimulation is also being considered for managing other psychiatric disorders, such as depression, ${ }^{89}$ Tourette syndrome ${ }^{82}$ and others discussed in this issue. Because of the potential for rational target development based on pathological circuits, DBS stands at the crossroads between advancing the understanding of the disordered networks underlying disease and treating those patients in whom all other treatment options have failed.

Given the increasingly well-characterized disordered brain circuits in individuals with autism, especially the circuits involved in socioemotional processing, it appears logical that addressing a faulty node of these circuits with DBS may provide relief for the most severe, problematic forms of autism. So far, 3 cases of DBS treatment for managing severe autism have been reported (Table 1). ${ }^{86,88}$ All 3 patients were experiencing prominent, life-threatening SIB that was not alleviated by multiple trials of antipsychotic medication. A 17-year-old male received DBS of the bilateral anterior limb of the internal capsule and of the globus pallidus internus. ${ }^{86}$ A 19 -year-old female received bilateral DBS to the globus pallidus internus only, ${ }^{86}$ and a male, at age 13 years, received DBS to the BLA bilaterally. ${ }^{88}$ In the first patient, some improvement in SIB was observed over the first 6 months, but the patient's condition gradually deteriorated back to his original baseline. However, in the latter 2 patients, at follow-ups 13 and 24 months post-DBS, respectively, SIB had improved to the point where it was no longer life threatening.

The choice of targets for the first 2 patients was based on viewing SIB from the perspective of stereotypical, compulsive behaviors, and thus the intervention targeted motor regions. For the third patient, the BLA was chosen because of its role in rage, fear, and social processing. ${ }^{88}$ Interestingly, in response to BLA DBS, the third patient experienced improvement in the social domain as well. Some subjectively noted improvements included single-word utterances in proper context, significantly decreased social anxiety, sleep improvement, and enjoyment of music, all of which were not achievable before DBS. ${ }^{88}$ Because of these social improvements, this patient's progress suggests the possibility of BLA being a target for the treatment of severe autism, with the caveat that this suggestion is based on a single report.

\section{Basolateral Nucleus DBS: Mechanistic and Technical Considerations}

As mentioned above, the BLA receives multisensory afferents and communicates with other amygdalar nuclei, the ventral striatum, and with PFC centers (such as the $\mathrm{OFC}, \mathrm{ACC}$, and mPFC) involved in social cognition and decision making. As such, the BLA may perform a "gate keeper" function, ${ }^{88}$ where information is processed within the relevance-detection and stimulus-valuation framework that has been theorized to characterize amygdalar func-

TABLE 1. Three reported cases of DBS treatment of severe autism phenotypes and their outcomes

\begin{tabular}{|c|c|c|c|c|c|}
\hline Authors \& Year & $\begin{array}{l}\text { Age (yrs), } \\
\text { Sex }\end{array}$ & Presentation & DBS Target† & DBS Parameters & Outcome \\
\hline \multicolumn{6}{|l|}{ Sturm et al., 2013} \\
\hline Case 1 & $13, M$ & $\begin{array}{l}\text { Life-threatening SIB, nonverbal } \\
\text { phenotype, \& profound so- } \\
\text { cial cognition deficits }\end{array}$ & BLA§ & $\begin{array}{l}120 \text {-msec pulse width, } 130-\mathrm{Hz} \text { fre- } \\
\text { quency, \& } 2 \text { to } 6.5-\mathrm{V} \text { amplitude }\end{array}$ & $\begin{array}{l}\text { Decrease in SIB \& improvement } \\
\text { in social cognition }\end{array}$ \\
\hline \multicolumn{6}{|l|}{$\begin{array}{l}\text { Stocco \& Baizabal- } \\
\text { Carvallo, } 2014\end{array}$} \\
\hline Case 2 ף & $19, \mathrm{~F}$ & $\begin{array}{l}\text { Refractory SIB, motor stereo- } \\
\text { typies, \& dystonia due to an- } \\
\text { tipsychotics }\end{array}$ & GPi & $\begin{array}{l}\text { 120-msec pulse width, } 80-\mathrm{Hz} \text { fre- } \\
\text { quency, \& bipolar 3.3-V ampli- } \\
\text { tude }\end{array}$ & $\begin{array}{l}\text { Decreased stereotypies \& de- } \\
\text { creased dystonia }\end{array}$ \\
\hline Case 3 & $17, \mathrm{M}$ & $\begin{array}{l}\text { Refractory SIB, motor stereo- } \\
\text { typies, \& intellectual dis- } \\
\text { ability }\end{array}$ & GPi \& ALIC & $\begin{array}{l}\text { 210-msec pulse width, } 100-\mathrm{Hz} \text { fre- } \\
\text { quency, \& bipolar 2.5-V ampli- } \\
\text { tude }\end{array}$ & $\begin{array}{l}\text { Transiently decreased in SIB \& } \\
\text { stereotypies, but then re- } \\
\text { turned to initial baseline after } \\
6 \text { mos }\end{array}$ \\
\hline
\end{tabular}

ALIC = anterior limb of internal capsule; GPi = globus pallidus internus.

* Age at surgery.

$\dagger$ All targets were bilateral.

$\ddagger$ At follow-up; the length of the follow-up periods was 24 months for Sturm et al. and 13 months for Stocco and Baizabal-Carvallo.

$\S$ Electrode contacts were in multiple amygdalar nuclei and in supra-amygdaloid white matter. Various permutations were tried, but only stimulation of BLA alone produced the noted symptom improvement.

I The patient had monosomy $2 q$ and trisomy $20 p$, but presented an autistic phenotype. 
tion. Specifically, stimuli from all modalities are analyzed and, if deemed significant on the basis of previous memory and reward learning, are imparted with emotional valence. This information is then transmitted to cortical centers to influence behavior. In the previously mentioned case of the patient treated with BLA DBS, improvements in both SIB and social cognition were noted. According to the extensive research into the disordered neural networks in people with autism, these symptoms appear to be the products of widespread network dysfunction involving frontotemporal regions, primary sensory cortices, basal ganglia, cerebellum, and others. However, because of the apparent role of the amygdala in integrating information across modalities, extracting significance from this information, and communicating with the other brain regions, the amygdala appears to be crucial in the pathogenesis of the major features of autism, including those of severe autism.

Given the afferent and efferent projections of the BLA, along with the therapeutic relief observed in the patient who received BLA DBS, the BLA may represent a critical substructure within the amygdala for treating severe autism. Given the aforementioned electrophysiological disturbances, symptom improvement after BLA DBS may in part be facilitated by a stimulation-induced reset of oscillatory activity. Mechanistically, this possibility may entail altering gamma-band activity such that proper phase locking between the amygdala and several higher-order socioemotional network regions occurs. Clearly, however, resolving the details of BLA DBS function necessitates further study.

As noted in their case report of BLA DBS, the authors encountered difficulties with surgical planning because of an anatomical heterogeneity of the amygdalar nuclei. ${ }^{88}$ Because amygdalar nuclei are grouped according to general anatomical region rather than by precisely defined locations, it may be difficult to find the best location for symptom relief in a given individual. In their placement of DBS electrodes, the authors attempted to cover multiple amygdalar regions, including central and superficial nuclei and supra-amygdaloid white matter projections, and tested multiple electrode contact combinations. Stimulation of the BLA alone was the only approach that exhibited therapeutic benefit. Although BLA targeting may be difficult, given the anatomical heterogeneity of the amygdala, the use of patient MRI data with template atlases can facilitate surgical planning. ${ }^{88}$

The ethical concerns of DBS for autism are of paramount importance. ${ }^{34,81}$ Careful patient selection should be of utmost priority because DBS is intended for those patients in whom autism symptoms are refractory to medical management. In autism, a heterogeneous disorder with nonstandard pharmacotherapy, patient selection will likely require even more nuanced attention, as the definition of "refractory" may be open to interpretation. Moreover, expectations of outcomes must be moderated, especially for caretakers. Sturm and colleagues, in their planning of BLA DBS, noted a careful and informative conversation with the patient's caretakers ${ }^{88}$ In particular, during the conversation, the authors stressed the experimental nature of the therapy. It is also important to note that DBS is not being proposed as a "cure for autism" or as a replacement for any form of early intervention, whether it be behavioral or pharmacological. It is in some regard a last-resort effort, especially in the current state of our understanding of autism where the pathological circuitry of the disorder is still awaiting further elucidation.

Despite these issues, DBS represents a promising therapeutic avenue for SIB and social deficits in severe autism. These symptoms can be life threatening for the patient and extremely challenging for the caretaker. Significant distress may arise among both if medical management fails to control these symptoms. In the absence of other therapeutic interventions for these patients, DBS may provide a therapeutic approach grounded in rational targeting of pathological circuitry. On the basis of findings from neuroimaging and electrophysiological studies characterizing the pathophysiology of autism, we suggest the BLA as a target warranting further study for therapeutic neuromodulation in patients with severe autism.

\section{Conclusions}

In summary, autism is a heterogeneous disorder. It is characterized by neurodevelopmental abnormalities that originate from dysfunctional genes involved in synaptogenesis and axon dynamics and from various environmental influences that have become increasingly well characterized. Structural studies along the developmental timeline have revealed a characteristic pattern of early overgrowth of brain regions such as PFC centers and of medial temporal lobe structures such as the amygdala. In addition, diffusion tensor imaging studies have shown impaired integrity of white matter structures involved in socioemotional processing such as the UF and AF. Moreover, functional neuroimaging and electrophysiological studies have demonstrated disordered activity in restingstate neural networks and abnormal task-based activation of these networks.

Among these abnormalities, the amygdala appears to be a key node involved in socioemotional deficits. Recent studies have begun to define the amygdala as an integrator of multisensory information and a detector of biological relevance and emotional valuation. Because social cognition depends on such detection and valuation, along with the fact that the amygdala consistently shows abnormalities in individuals with autism, this structure appears to be a logical target for neuromodulation to manage severe autism.

The most severe cases of autism are characterized by a complete lack of language development and by SIB, the latter of which can be refractory to medical management. Deep brain stimulation of the BLA represents a potential therapeutic intervention and has previously been used in one case report of severe autism. Based on its connections, the BLA may be the substructure of the amygdala in which integration and valuation are performed, and its dysfunction may underlie the SIB and deficits in social cognition observed in individuals with autism. Therefore, BLA DBS deserves careful study of its potential as a therapeutic intervention in patients with severe autism who are experiencing crippling, life-threatening symptoms with no pharmacotherapeutic recourse. 


\section{References}

1. Adler BA, Wink LK, Early M, Shaffer R, Minshawi N, McDougle CJ, et al: Drug-refractory aggression, self-injurious behavior, and severe tantrums in autism spectrum disorders: a chart review study. Autism 19:102-106, 2015

2. Adolphs R: What does the amygdala contribute to social cognition? Ann N Y Acad Sci 1191:42-61, 2010

3. Adolphs R, Gosselin F, Buchanan TW, Tranel D, Schyns P, Damasio AR: A mechanism for impaired fear recognition after amygdala damage. Nature 433:68-72, 2005

4. Adolphs R, Tranel D, Buchanan TW: Amygdala damage impairs emotional memory for gist but not details of complex stimuli. Nat Neurosci 8:512-518, 2005

5. Alexander GE, DeLong MR, Strick PL: Parallel organization of functionally segregated circuits linking basal ganglia and cortex. Annu Rev Neurosci 9:357-381, 1986

6. Ameis SH, Catani M: Altered white matter connectivity as a neural substrate for social impairment in Autism Spectrum Disorder. Cortex 62:158-181, 2014

7. Amodio DM, Frith CD: Meeting of minds: the medial frontal cortex and social cognition. Nat Rev Neurosci 7:268-277, 2006

8. Ashwin C, Baron-Cohen S, Wheelwright S, O'Riordan M, Bullmore ET: Differential activation of the amygdala and the 'social brain' during fearful face-processing in Asperger Syndrome. Neuropsychologia 45:2-14, 2007

9. Assaf M, Jagannathan K, Calhoun VD, Miller L, Stevens MC, Sahl R, et al: Abnormal functional connectivity of default mode sub-networks in autism spectrum disorder patients. Neuroimage 53:247-256, 2010

10. Bauman ML, Kemper TL: Neuroanatomic observations of the brain in autism: a review and future directions. Int J Dev Neurosci 23:183-187, 2005

11. Bosch J, Van Dyke C, Smith SM, Poulton S: Role of medical conditions in the exacerbation of self-injurious behavior: an exploratory study. Ment Retard 35:124-130, 1997

12. Boser K, Higgins S, Fetherston A, Preissler MA, Gordon B: Semantic fields in low-functioning autism. J Autism Dev Disord 32:563-582, 2002

13. Buzsáki G: Rhythms of the Brain. Oxford: Oxford University Press, 2011

14. Bzdok D, Langner R, Schilbach L, Engemann DA, Laird AR, Fox PT, et al: Segregation of the human medial prefrontal cortex in social cognition. Front Hum Neurosci 7:232, 2013

15. Carlson GC: Glutamate receptor dysfunction and drug targets across models of autism spectrum disorders. Pharmacol Biochem Behav 100:850-854, 2012

16. Castelli F, Frith C, Happé F, Frith U: Autism, Asperger syndrome and brain mechanisms for the attribution of mental states to animated shapes. Brain 125:1839-1849, 2002

17. Catani M, Dell'acqua F, Thiebaut de Schotten M: A revised limbic system model for memory, emotion and behaviour. Neurosci Biobehav Rev 37:1724-1737, 2013

18. Cauda F, Costa T, Palermo S, D'Agata F, Diano M, Bianco F, et al: Concordance of white matter and gray matter abnormalities in autism spectrum disorders: a voxel-based metaanalysis study. Hum Brain Mapp 35:2073-2098, 2014

19. Chang J, Gilman SR, Chiang AH, Sanders SJ, Vitkup D: Genotype to phenotype relationships in autism spectrum disorders. Nat Neurosci 18:191-198, 2015

20. Coben R, Clarke AR, Hudspeth W, Barry RJ: EEG power and coherence in autistic spectrum disorder. Clin Neurophysiol 119:1002-1009, 2008

21. Cornew L, Roberts TPL, Blaskey L, Edgar JC: Resting-state oscillatory activity in autism spectrum disorders. J Autism Dev Disord 42:1884-1894, 2012

22. Dalton KM, Nacewicz BM, Johnstone T, Schaefer HS, Gernsbacher MA, Goldsmith HH, et al: Gaze fixation and the neural circuitry of face processing in autism. Nat Neurosci 8:519-526, 2005
23. Devine DP: Self-injurious behaviour in autistic children: a neuro-developmental theory of social and environmental isolation. Psychopharmacology (Berl) 231:979-997, 2014

24. Di Martino A, Ross K, Uddin LQ, Sklar AB, Castellanos FX, Milham MP: Functional brain correlates of social and nonsocial processes in autism spectrum disorders: an activation likelihood estimation meta-analysis. Biol Psychiatry 65:63-74, 2009

25. Di Martino A, Yan CG, Li Q, Denio E, Castellanos FX, Alaerts K, et al: The autism brain imaging data exchange: towards a large-scale evaluation of the intrinsic brain architecture in autism. Mol Psychiatry 19:659-667, 2014

26. Duerden EG, Mak-Fan KM, Taylor MJ, Roberts SW: Regional differences in grey and white matter in children and adults with autism spectrum disorders: an activation likelihood estimate (ALE) meta-analysis. Autism Res 5:49-66, 2012

27. Ecker C, Suckling J, Deoni SC, Lombardo MV, Bullmore ET, Baron-Cohen S, et al: Brain anatomy and its relationship to behavior in adults with autism spectrum disorder: a multicenter magnetic resonance imaging study. Arch Gen Psychiatry 69:195-209, 2012

28. Edgar JC, Khan SY, Blaskey L, Chow VY, Rey M, Gaetz W, et al: Neuromagnetic oscillations predict evoked-response latency delays and core language deficits in autism spectrum disorders. J Autism Dev Disord 45:395-405, 2015

29. Fried I, MacDonald KA, Wilson CL: Single neuron activity in human hippocampus and amygdala during recognition of faces and objects. Neuron 18:753-765, 1997

30. Frith U, Frith CD: Development and neurophysiology of mentalizing. Philos Trans R Soc Lond B Biol Sci 358:459-473, 2003

31. Geschwind DH: Genetics of autism spectrum disorders. Trends Cogn Sci 15:409-416, 2011

32. Gottfried JA, O'Doherty J, Dolan RJ: Encoding predictive reward value in human amygdala and orbitofrontal cortex. Science 301: 1104-1107, 2003

33. Gotts SJ, Simmons WK, Milbury LA, Wallace GL, Cox RW, Martin A: Fractionation of social brain circuits in autism spectrum disorders. Brain 135:2711-2725, 2012

34. Grant RA, Halpern CH, Baltuch GH, O'Reardon JP, Caplan A: Ethical considerations in deep brain stimulation for psychiatric illness. J Clin Neurosci 21:1-5, 2014

35. Hadjikhani N, Joseph RM, Snyder J, Tager-Flusberg H: Abnormal activation of the social brain during face perception in autism. Hum Brain Mapp 28:441-449, 2007

36. Hahamy A, Behrmann M, Malach R: The idiosyncratic brain: distortion of spontaneous connectivity patterns in autism spectrum disorder. Nat Neurosci 18:302-309, 2015

37. Hamani C, Pilitsis J, Rughani AI, Rosenow JM, Patil PG, Slavin KS, et al.: Deep brain stimulation for obsessivecompulsive disorder: systematic review and evidence-based guideline sponsored by the American Society for Stereotactic and Functional Neurosurgery and the Congress of Neurological Surgeons (CNS) and endorsed by the CNS and American Association of Neurological Surgeons. Neurosurgery 75:327-333, 2014

38. Heilbronner SR, Haber SN: Frontal cortical and subcortical projections provide a basis for segmenting the cingulum bundle: implications for neuroimaging and psychiatric disorders. J Neurosci 34:10041-10054, 2014

39. Holtzheimer PE, Kelley ME, Gross RE, Filkowski MM, Garlow SJ, Barrocas A, et al: Subcallosal cingulate deep brain stimulation for treatment-resistant unipolar and bipolar depression. Arch Gen Psychiatry 69:150-158, 2012

40. Huang MX, Theilmann RJ, Robb A, Angeles A, Nichols S, Drake A, et al: Integrated imaging approach with MEG and DTI to detect mild traumatic brain injury in military and civilian patients. J Neurotrauma 26:1213-1226, 2009

41. Huerta M, Bishop SL, Duncan A, Hus V, Lord C: Applica- 
tion of DSM-5 criteria for autism spectrum disorder to three samples of children with DSM-IV diagnoses of pervasive developmental disorders. Am J Psychiatry 169:1056-1064, 2012

42. Idring S, Rai D, Dal H, Dalman C, Sturm H, Zander E, et al: Autism spectrum disorders in the Stockholm Youth Cohort: design, prevalence and validity. PLoS ONE 7:e41280, 2012

43. Jung HH, Kim CH, Chang JH, Park YG, Chung SS, Chang JW: Bilateral anterior cingulotomy for refractory obsessivecompulsive disorder: Long-term follow-up results. Stereotact Funct Neurosurg 84:184-189, 2006

44. Kann O, Papageorgiou IE, Draguhn A: Highly energized inhibitory interneurons are a central element for information processing in cortical networks. J Cereb Blood Flow Metab 34:1270-1282, 2014

45. Kanner L: Autistic disturbances of affective contact. Acta Paedopsychiatr 35:100-136, 1968

46. Kapp BS, Frysinger RC, Gallagher M, Haselton JR: Amygdala central nucleus lesions: effect on heart rate conditioning in the rabbit. Physiol Behav 23:1109-1117, 1979

47. Kennedy DP, Redcay E, Courchesne E: Failing to deactivate: resting functional abnormalities in autism. Proc Natl Acad Sci US A 103:8275-8280, 2006

48. Kier EL, Staib LH, Davis LM, Bronen RA: MR imaging of the temporal stem: anatomic dissection tractography of the uncinate fasciculus, inferior occipitofrontal fasciculus, and Meyer's loop of the optic radiation. AJNR Am J Neuroradiol 25:677-691, 2004

49. Kim YS, Leventhal BL, Koh YJ, Fombonne E, Laska E, Lim EC, et al: Prevalence of autism spectrum disorders in a total population sample. Am J Psychiatry 168:904-912, 2011

50. Lai MC, Lombardo MV, Baron-Cohen S: Autism. Lancet 383:896-910, 2014

51. Lai MC, Lombardo MV, Suckling J, Ruigrok ANV, Chakrabarti B, Ecker C, et al: Biological sex affects the neurobiology of autism. Brain 136:2799-2815, 2013

52. Le JF, Lohr WD: Aggression and self-injury in a patient with severe autism. Pediatr Ann 41:1-3, 2012

53. LeDoux JE, Iwata J, Cicchetti P, Reis DJ: Different projections of the central amygdaloid nucleus mediate autonomic and behavioral correlates of conditioned fear. J Neurosci 8:2517-2529, 1988

54. Likhtik E, Stujenske JM, Topiwala MA, Harris AZ, Gordon JA: Prefrontal entrainment of amygdala activity signals safety in learned fear and innate anxiety. Nat Neurosci 17:106-113, 2014

55. Luzzani S, Macchini F, Valadè A, Milani D, Selicorni A: Gastroesophageal reflux and Cornelia de Lange syndrome: typical and atypical symptoms. Am J Med Genet A 119A:283-287, 2003

56. Mailis A: Compulsive targeted self-injurious behaviour in humans with neuropathic pain: a counterpart of animal autotomy? Four case reports and literature review. Pain 64:569-578, 1996

57. Marco EJ, Hinkley LBN, Hill SS, Nagarajan SS: Sensory processing in autism: a review of neurophysiologic findings. Pediatr Res 69:48R-54R, 2011

58. Martínez-Sanchis S: Neurobiological foundations of multisensory integration in people with autism spectrum disorders: the role of the medial prefrontal cortex. Front Hum Neurosci 8:970, 2014

59. Mehta MV, Gandal MJ, Siegel SJ: mGluR5-antagonist mediated reversal of elevated stereotyped, repetitive behaviors in the VPA model of autism. PLoS ONE 6:e26077, 2011

60. Meletti S, Cantalupo G, Benuzzi F, Mai R, Tassi L, Gasparini $\mathrm{E}$, et al: Fear and happiness in the eyes: an intra-cerebral event-related potential study from the human amygdala. Neuropsychologia 50:44-54, 2012

61. Minshew NJ, Keller TA: The nature of brain dysfunction in autism: functional brain imaging studies. Curr Opin Neurol 23:124-130, 2010

62. Murray RJ, Brosch T, Sander D: The functional profile of the human amygdala in affective processing: insights from intracranial recordings. Cortex 60:10-33, 2014

63. Newell KM, Challis JH, Boros RL, Bodfish JW: Further evidence on the dynamics of self-injurious behaviors: impact forces and limb motions. Am J Ment Retard 107:60-68, 2002

64. Newell KM, Sprague RL, Pain MT, Deutsch KM, Meinhold P: Dynamics of self-injurious behaviors. Am J Ment Retard 104:11-21, 1999

65. Orekhova EV, Stroganova TA, Nygren G, Tsetlin MM, Posikera IN, Gillberg C, et al: Excess of high frequency electroencephalogram oscillations in boys with autism. Biol Psychiatry 62:1022-1029, 2007

66. Palmen SJMC, van Engeland H, Hof PR, Schmitz C: Neuropathological findings in autism. Brain 127:2572-2583, 2004

67. Peebles KA, Price TJ: Self-injurious behaviour in intellectual disability syndromes: evidence for aberrant pain signalling as a contributing factor. J Intellect Disabil Res 56:441-452, 2012

68. Pellicano E, Maybery M, Durkin K, Maley A: Multiple cognitive capabilities/deficits in children with an autism spectrum disorder: "weak" central coherence and its relationship to theory of mind and executive control. Dev Psychopathol 18:77-98, 2006

69. Port RG, Gandal MJ, Roberts TPL, Siegel SJ, Carlson GC: Convergence of circuit dysfunction in ASD: a common bridge between diverse genetic and environmental risk factors and common clinical electrophysiology. Front Cell Neurosci 8:414, 2014

70. Pugliese L, Catani M, Ameis S, Dell'Acqua F, Thiebaut de Schotten M, Murphy C, et al: The anatomy of extended limbic pathways in Asperger syndrome: a preliminary diffusion tensor imaging tractography study. Neuroimage 47:427-434, 2009

71. Raymond GV, Bauman ML, Kemper TL: Hippocampus in autism: a Golgi analysis. Acta Neuropathol 91:117-119, 1996

72. Richards C, Oliver C, Nelson L, Moss J: Self-injurious behaviour in individuals with autism spectrum disorder and intellectual disability. J Intellect Disabil Res 56:476-489, 2012

73. Roberts TPL, Khan SY, Rey M, Monroe JF, Cannon K, Blaskey L, et al: MEG detection of delayed auditory evoked responses in autism spectrum disorders: towards an imaging biomarker for autism. Autism Res 3:8-18, 2010

74. Rubenstein JLR, Merzenich MM: Model of autism: increased ratio of excitation/inhibition in key neural systems. Genes Brain Behav 2:255-267, 2003

75. Rutishauser U, Tudusciuc O, Wang S, Mamelak AN, Ross IB, Adolphs R: Single-neuron correlates of atypical face processing in autism. Neuron 80:887-899, 2013

76. Sah P, Faber ESL, Lopez De Armentia M, Power J: The amygdaloid complex: anatomy and physiology. Physiol Rev 83:803-834, 2003

77. Sahyoun CP, Belliveau JW, Soulières I, Schwartz S, Mody $\mathrm{M}$ : Neuroimaging of the functional and structural networks underlying visuospatial vs. linguistic reasoning in high-functioning autism. Neuropsychologia 48:86-95, 2010

78. Sander D, Grafman J, Zalla T: The human amygdala: an evolved system for relevance detection. Rev Neurosci 14:303-316, 2003

79. Sato W, Kochiyama T, Uono S, Matsuda K, Usui K, Inoue Y, et al: Rapid amygdala gamma oscillations in response to eye gaze. PLoS ONE 6:e28188, 2011

80. Scherf KS, Elbich D, Minshew N, Behrmann M: Individual differences in symptom severity and behavior predict neural activation during face processing in adolescents with autism. Neuroimage Clin 7:53-67, 2015 
81. Schermer M: Ethical issues in deep brain stimulation. Front Integr Neurosci 5:17, 2011

82. Schrock LE, Mink JW, Woods DW, Porta M, Servello D, Visser-Vandewalle V, et al: Tourette syndrome deep brain stimulation: A review and updated recommendations. Mov Disord [epub ahead of print], 2014

83. Schumann CM, Hamstra J, Goodlin-Jones BL, Lotspeich LJ, Kwon $\mathrm{H}$, Buonocore $\mathrm{MH}$, et al: The amygdala is enlarged in children but not adolescents with autism; the hippocampus is enlarged at all ages. J Neurosci 24:6392-6401, 2004

84. Spezio ML, Huang PY, Castelli F, Adolphs R: Amygdala damage impairs eye contact during conversations with real people. J Neurosci 27:3994-3997, 2007

85. Stigler KA, McDougle CJ: Pharmacotherapy of irritability in pervasive developmental disorders. Child Adolesc Psychiatr Clin N Am 17:739-752, vii-viii, 2008

86. Stocco A, Baizabal-Carvallo JF: Deep brain stimulation for severe secondary stereotypies. Parkinsonism Relat Disord 20:1035-1036, 2014

87. Stoner R, Chow ML, Boyle MP, Sunkin SM, Mouton PR, Roy S, et al: Patches of disorganization in the neocortex of children with autism. N Engl J Med 370:1209-1219, 2014

88. Sturm V, Fricke O, Bührle CP, Lenartz D, Maarouf M, Treuer $\mathrm{H}$, et al: DBS in the basolateral amygdala improves symptoms of autism and related self-injurious behavior: a case report and hypothesis on the pathogenesis of the disorder. Front Hum Neurosci 6:341, 2013

89. Taghva AS, Malone DA, Rezai AR: Deep brain stimulation for treatment-resistant depression. World Neurosurg 80:S27. e17-S27.e24, 2013

90. Tottenham N, Hertzig ME, Gillespie-Lynch K, Gilhooly T, Millner AJ, Casey BJ: Elevated amygdala response to faces and gaze aversion in autism spectrum disorder. Soc Cogn Affect Neurosci 9:106-117, 2014
91. Van Overwalle F: Social cognition and the brain: a metaanalysis. Hum Brain Mapp 30:829-858, 2009

92. Veinante P, Yalcin I, Barrot M: The amygdala between sensation and affect: a role in pain. J Mol Psychiatry 1:9, 2013

93. Voineagu I, Wang X, Johnston P, Lowe JK, Tian Y, Horvath $\mathrm{S}$, et al: Transcriptomic analysis of autistic brain reveals convergent molecular pathology. Nature 474:380-384, 2011

94. Vuilleumier P: How brains beware: neural mechanisms of emotional attention. Trends Cogn Sci 9:585-594, 2005

95. Zahorodny W, Shenouda J, Howell S, Rosato NS, Peng B, Mehta U: Increasing autism prevalence in metropolitan New Jersey. Autism 18:117-126, 2014

96. Zalla T, Sperduti M: The amygdala and the relevance detection theory of autism: an evolutionary perspective. Front Hum Neurosci 7:894, 2013

97. Zikopoulos B, Barbas H: Changes in prefrontal axons may disrupt the network in autism. J Neurosci 30:14595-14609, 2010

\section{Author Contributions}

Conception and design: all authors. Analysis and interpretation of data: Sinha. Drafting the article: Sinha. Critically revising the article: all authors. Reviewed submitted version of manuscript: all authors. Statistical analysis: McGovern.

\section{Correspondence}

Sameer A. Sheth, Department of Neurological Surgery, Columbia University Medical Center, 710 W. 168th St., New York, NY 10032.email: ss4451@cumc.columbia.edu. 